\title{
Evaluating the spatial and temporal patterns of the severe fever thrombocytopenia syndrome in Republic of Korea
}

\author{
Seongwoo Park, ${ }^{1,2}$ Hae-Sung Nam, ${ }^{3}$ Baeg-Ju Na ${ }^{4}$ \\ ${ }^{1}$ Department of Public Health, Chungnam National University Graduate School, Daejeon; ${ }^{2}$ Division of \\ Climate Change and Health Protection, Korea Disease Control and Prevention Agency (KDCA), \\ Chungcheongbuk-do; ${ }^{3}$ Department of Preventive Medicine, Chungnam National University College of \\ Medicine, Daejeon; ${ }^{4}$ Graduate School of Urban Public Health, University of Seoul, Seoul, Republic of Korea
}

\begin{abstract}
Severe fever with thrombocytopenia syndrome (SFTS) is a new infectious disease with a high mortality rate and increased incidence in Republic of Korea since the first case was reported in 2013. The average mortality rate varies by region and year but remains high in Asia. This study aimed to evaluate the spatial and temporal patterns of SFTS cases reported to the national Disease Control and Prevention Agency (KDCA). We analysed the spatial and temporal distribution of SFTS and observed changes in areas vulnerable to the disease. We analysed data concerning 1086 con-
\end{abstract}

Correspondence: Baeg-Ju Na, Graduate School of Urban Public Health, University of Seoul, 163, Seoulsiripdae-ro, Dongdaemun-gu, Seoul, Republic of Korea.

Tel.: +82.10.3606.5135 - Fax: +82.2 .6490 .6754 .

E-mail: baegju.na@uos.ac.kr

Key words: Clusters; severe fever with thrombocytopenia syndrome; spatiotemporal; Republic of Korea.

Acknowledgements: we would like to thank Editage (www.editage.com) for English language editing. We also thank the anonymous reviewers for their insightful comments and great efforts to help improve our manuscript.

Ethical approval: this study used public data on severe febrile thrombocytopenia syndrome (SFTS) provided by the Korea Centers for Disease Control and Prevention (KDCA) and was conducted with the approval of the Chungnam National University Institutional Ethics Committee. As this was a retrospective study, the requirement for informed consent was waived as per Institutional Review Board approval (IRB no. 202012-SB-161-01).

Received for publication: 4 March 2021.

Revision received: 27 May 2021.

Accepted for publication: 27 May 2021.

CCopyright: the Author(s), 2021

Licensee PAGEPress, Italy

Geospatial Health 2021; 16:994

doi:10.4081/gh.2021.994

This article is distributed under the terms of the Creative Commons Attribution Noncommercial License (CC BY-NC 4.0) which permits any noncommercial use, distribution, and reproduction in any medium, provided the original author(s) and source are credited. firmed SFTS patients from 2013 to 2019 categorized according to the 247 district level administrative units. To better understand the epidemiology of SFTS, we carried out spatiotemporal analyses on a yearly basis and also calculated and mapped spatial clusters of domestic SFTS by global (regional) and local Moran's indices. To observe the annual changes in SFTS incidence rate, scan statistics for each city and district were calculated. The incidence rate showed significant clustering in specific regions, which reoccurred annually in some regions. In Republic of Korea, SFTS clusters have been expanding into the southern regions, with annual clusters concentrated between May and October. This pattern allows prediction of SFTS occurrences through spatiotemporal analysis, which makes it possible to guide measures of disease prevention.

\section{Introduction}

Severe fever with thrombocytopenia syndrome (SFTS) is a tick-borne infectious disease first identified in rural areas of Hunan and Hubei in central China in 2009 (You et al., 2020). During the initial epidemiological investigation in China, a group of patients with high fever, digestive symptoms and low platelet and white blood cell counts was observed. The causative agent for this disease was confirmed as the severe fever thrombocytopenia syndrome virus (SFTSV) belonging to the Bunyaviridae family (Yu et al., 2011). The clinical signs of SFTS include fever, thrombocytopenia, and leukopenia, accompanied by coldness, headache, lymphoedema, anorexia, nausea, muscle pain, diarrhoea, vomiting, abdominal pain, bleeding gums, and conjunctival opacity (Yu et al., 2011; Ding et al., 2013). Although numbers of cases and deaths are increasing, effective treatment will be difficult to establish (Oh et al., 2014).

Since its discovery, more than 13,000 SFTS cases have been reported worldwide, of which more than $8 \%$ have died (Miao et al., 2020). More than $90 \%$ of SFTS cases have occurred in China, while the highest annual growth rate was recently observed in Republic of Korea (Miao et al., 2020), where the disease is treated as a new notifiable infectious disease since 23 September 2013 (Seo et al., 2017). In Republic of Korea, the first outbreak of SFTS was reported in May 2013, and the primary vector found to be the longhorned tick, Haemaphysalis longicornis (KDCA, 2016). Suspected cases are reported to the national Centers for Disease Control and Prevention (KDCA) within 24 hours, where the data on confirmed SFTS patients are recorded in a dedicated database and epidemiological investigations subsequently conducted.

In China, most hospitalized patients with SFTS are outdoor workers and the epidemic period of SFTS is from March to 
November, with incidence rates peaking in June and July. So far, more than $50 \%$ of SFTS cases have occurred in Henan and Hubei provinces (Li et al., 2017). In Japan, the first death due to SFTS was registered in the fall of 2012 (Takahashi et al., 2014) and a case of infection with a virus related to SFTSV, the Heartland virus, was reported in the same year in the United States (McMullan et al., 2012). Although the average mortality rate varies by region and year, it has remained relatively high in Japan (27\%), Korea (23.3\%) and China (5.3\%-16.2\%) (Yun et al., 2020). According to China's national surveillance data covering 2011 to 2013, the number of patients with SFTS increased, while the number of deaths decreased (Liu et al., 2015). In this country, the disease mostly affects the elderly and farmers (Liu et al., 2015), while other epidemiologic characteristics, such as incidence, sex ratio, fatality rate, and seasonality vary considerably depending on the affected region, which could be due to local agricultural activities and the seasonal tick situation (Liu et al., 2015). Severe outcome of SFTSV infection has been found to be related to climatic factors such as altitude, temperature, precipitation and relative humidity, with the probability of occurrence increasing under certain conditions (Sun et al., 2020).

Since the initial symptoms are not distinguishable from other infectious diseases, the diagnosis is difficult. In addition, the clinical, management is challenging due to the lack of effective preventive measures and clear means of treatment (Yoo \& Heo, 2018). The fatality rate of SFTS differs across countries and regions, and there are regions with a fatality rate of $30 \%$ (Guo et al., 2016; Liu et al., 2015). On average, most patients died nine days after symptom onset, otherwise within 2 weeks (Yoo \& Heo, 2018). Patients with complications can be difficult to manage secondary to their rapid clinical deterioration. Often, these patients die from unspecified sepsis (Oh et al., 2014; Yoo \& Heo, 2018).

Infectious diseases such as SFTS appear repeatedly in a specific space and time; hence, areas with a high risk of occurrence should be identified to inform and initiate rapid prevention measures. The purpose of this study was to investigate the temporal and spatial characteristics of SFTS in Republic of Korea. Specifically, the study investigated the clustering and spatiotemporal propagation patterns of SFTS.

\section{Materials and methods}

We analysed data of 1,086 SFTS patients, by administrative district, based on the data collected by the KDCA over the period 2013-2019. A cross table of the major characteristics of SFTS patients including deaths by season, region, sex and age was created, and statistical significance was evaluated using chi-square and fish exact tests. Global and local Moran's I and spatiotemporal scan statistics were used to evaluate the range of temporal and spatial clustering (clusters or hotspots) of domestic SFTS and the geographical risk patterns. Such a result can intuitively convey the spatial location of the disease cluster through geographic information systems (GIS). For spatial analysis, the cumulative incidence of SFTS per 100,000 people per year was calculated using the resident registered population for each of the 247 administrative districts as follows:

$$
\text { Cumulative incidence }=\frac{\text { SFTS cases per year and district }}{\text { Resident population per year and district }} \times 100,000
$$

First, Moran's I was used to determine the spatial autocorrelation of SFTS occurrence in each city, county and district during the study period. Moran's $I$ values range from -1 to 1 where 0 equal to normal distribution, positive values indicating clustered distributions or spatial autocorrelation and negative values indicating scattered distributions. Global Moran's $I$ was used for the entire space and Local Moran's I regional distributions. The concept of local indicators of special assistance (LISA) analysis was developed by Anselin (1995). LISA calculates the value of Moran's $I$ according to administrative district for the entire study area, thus identifying spatial autocorrelations by administrative district that can be calculated as follows:

$$
I_{i}=\frac{\left(x_{i}-x^{*}\right) \sum_{j} w_{i j}\left(x_{j}-x^{*}\right)}{\sum_{i}\left(x_{i}-x^{*}\right)^{2} / n}
$$

where $x_{i}$ is the value of $x$ in spatial unit $i ; x^{*}$ the mean of $x ; n$ the number of spatial units; and $w_{i j}$ the spatial weights matrix that measures the strength of the relationship between two spatial units. This formula generates values for local Moran's $I$ that range from -1 (perfect negative spatial autocorrelation) to +1 (perfect positive spatial autocorrelation) (Andresen, 2011).

\section{Cluster classification}

The SFTS clusters were divided into four groups: High-high $(\mathrm{HH})$ clusters $=$ high values in a high-value neighbourhood areas; low-low (LL) clusters = low values in a low-value neighbourhood areas; high low (HL) spatial outlier = a high value in a low-value neighbourhood; and low-high $(\mathrm{LH})=$ a low value in a high-value neighbourhood

In this study, SaTScan ${ }^{\mathrm{TM}}$ Software (Kulldorff, 2018) was used to evaluate the occurrence pattern of spatio-temporal clusters of SFTS, according to spatiotemporal factors. The spatio-temporal scan statistics using SaTScan were based on the Poisson model and were used to identify cluster areas with a statistically significant SFTS occurrence rate. Seasons were divided into spring (MarchMay), summer (June-August), autumn (September-November), and winter (December of the year and January and February of the following year).

Kulldorff's SaTScan gradually increases the size of each regional unit to create a hierarchical window that overlaps each other and calculates the log likelihood ratio (LLR) for all windows and the maximum test statistic. The scan statistics were tested for significance using the Monte Carlo approach, which randomly generates a large number of datasets under the null hypothesis (i.e. the risks of SFTS are the same in the units of space). Then, we calculated the test statistic for each data set to determine significance by checking the rank of the values of the test statistic calculated from the actual data. A simulation with 999 permutations was performed to ensure adequate power for defining the clusters (Kulldorff, 2018), which means that if the value of the test statistic calculated from the identified cluster falls within the top $10 \%$, the cluster should be considered significant (Han \& Lee, 2016).

The relative risk (RR) ratio, given by dividing the predicted risk value within the cluster by the predicted risk value outside the cluster, was calculated for each administrative district using the number of SFTS occurrences and population data during the study period. For example, an RR value of 10 for an administrative district means that the population in that area is 10 times more likely to be exposed to SFTS. 


\section{Results}

The number of confirmed SFTS patients reported to the KDCA from 2013 to 2019 was 1086, including 214 dead, which gives a fatality rate of $19.7 \%$ (Table 1 ). Although the number of male and female cases was equal $(n=543)$, there were more deaths among the women $(54.7 \%)$ than the men $(45.3 \%)$. However, the difference was not statistically significant $(\mathrm{P}=0.147)$.

In terms of temporal occurrence there was a rising trend as seen in Figure 1. Out of the 36 cases of SFTS in the first year, 17 died. In 2019, that had changed to 223 cases and 41 deaths in 2019. Over the whole study period, most cases occurred between May and October $(n=1061,97.7 \%)$ and more frequent in summer $(\mathrm{n}=509,47.0 \%)$ and fall $(\mathrm{n}=493,45.3 \%)$, indicating a seasonal cycle. Indeed, the cumulative incidence of SFTS per 100,000 people per year in the 247 administrative districts showed an increasing trend and expansion into the eastern and southern region (Figure 2).

By age, $55.9 \%$ of all patients (607) were over 65 years, and the number of patients increased with age (Table 1 and Figure 1). The global spatial autocorrelation analysis of the annual incidences of SFTS through Global Moran's I significant clustering at the
Table 1. Spatiotemporal overview of patients and outcomes with respect to the severe fever with thrombocytopenia syndrome.

\begin{tabular}{|c|c|c|c|c|}
\hline Characteristic & $\begin{array}{c}\text { Non-fatal } \\
\text { No. (\%) }\end{array}$ & $\begin{array}{c}\text { Fatal } \\
\text { No. }(\%)\end{array}$ & $\begin{array}{c}\text { Total } \\
\text { No. }(\%)\end{array}$ & $\mathbf{P}$ \\
\hline $\begin{array}{l}\text { Season } \\
\text { - Spring } \\
\text { - Summer } \\
\text { - Fall } \\
\text { - Winter }\end{array}$ & $\begin{array}{c}59(6.8) \\
394(45.2) \\
418(47.9) \\
1(0.1)\end{array}$ & $\begin{array}{c}24(11.2) \\
115(53.7) \\
75(35.0) \\
0(0.0)\end{array}$ & $\begin{array}{c}83(7.6) \\
509(46.9) \\
493(45.4) \\
1(0.1)\end{array}$ & $<0.01$ \\
\hline $\begin{array}{l}\text { Region } \\
\text { - District } \\
\text { - City } \\
\text { - County }\end{array}$ & $\begin{array}{l}165(18.9) \\
299(34.3) \\
408(46.8)\end{array}$ & $\begin{array}{l}33(15.4) \\
87(40.7) \\
87(40.7)\end{array}$ & $\begin{array}{l}198(18.2) \\
502(46.2) \\
386(35.5)\end{array}$ & 0.181 \\
\hline $\begin{array}{l}\text { Sex } \\
\text { - Male } \\
\text { - Female }\end{array}$ & $\begin{array}{l}446(51.1) \\
426(48.9)\end{array}$ & $\begin{array}{c}97(45.3) \\
117(54.7)\end{array}$ & $\begin{array}{l}543(50) \\
543(50)\end{array}$ & 0.147 \\
\hline $\begin{array}{l}\text { Age } \\
-<65 \\
-\geq 65 \\
\end{array}$ & $\begin{array}{l}429(49.2) \\
443(50.8)\end{array}$ & $\begin{array}{c}50(23.4) \\
164(76.6)\end{array}$ & $\begin{array}{l}479(44.1) \\
607(55.9)\end{array}$ & $<0.01$ \\
\hline Total & $872(100)$ & $214(100)$ & $1086(100)$ & \\
\hline
\end{tabular}

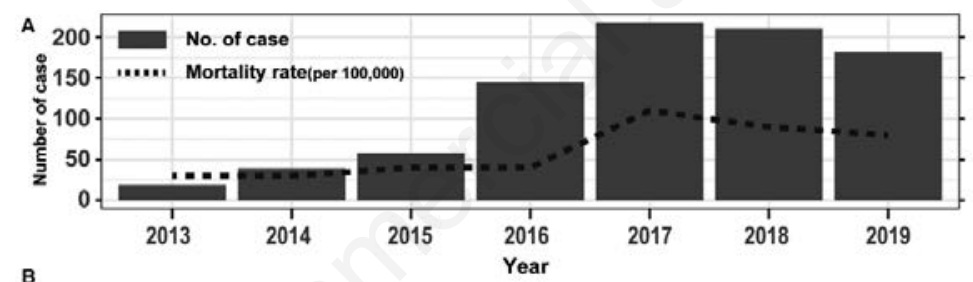

$$
\text { B }
$$
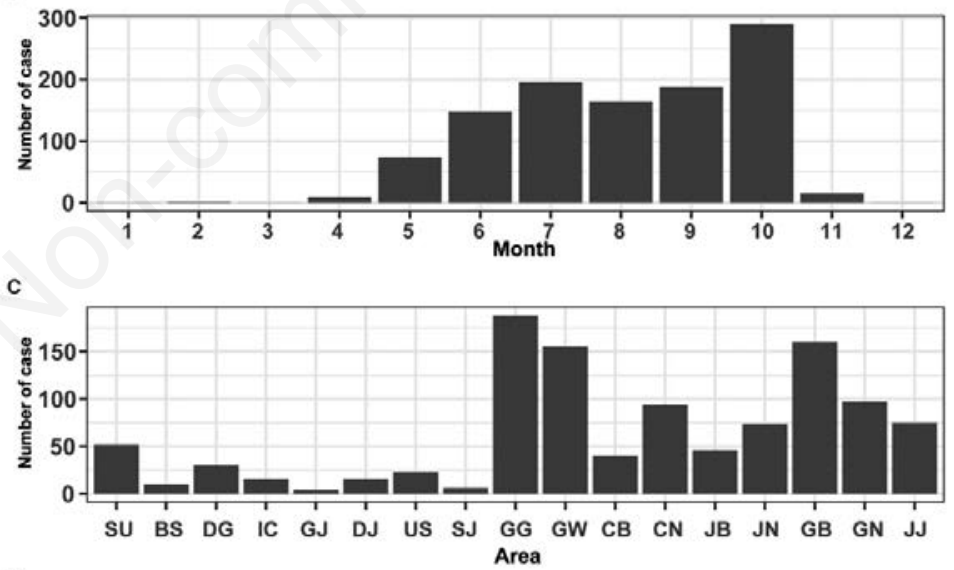

D

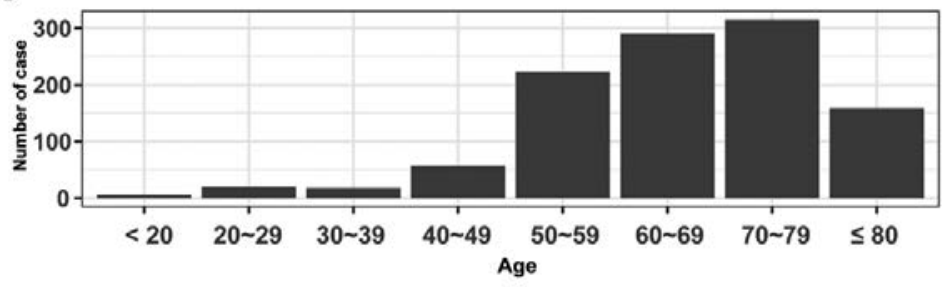

Figure 1. Distribution of patients with the severe fever with thrombocytopenia syndrome in Republic of Korea 2013-2019. A) year; B) month; C) area*; D) age. ${ }^{*} \mathrm{SU}=$ Seoul, BS=Busan, DG=Daegu, IC=Incheon, GJ=Gwangju, DJ=Daejeon, US=Ulsan, SJ=Sejong-si, GG=Gyeonggi-do, GW=Gangwon-do, CB=Chungcheongbuk-do, CN=Chungcheongnam-do, JB=Jeollabuk-do, JN=Jellanam-do, GB=Gyeongsangbuk-do, GN=Gyeongsangnam-do, JJ=Jeju-do. 
$\mathrm{P}=0.01$ level had positive $(+)$ values in all years. According to the spatial autocorrelation results, the early monitoring period (20132015) showed relatively low Moran's I values, while the more ones (2016-2019) had relatively higher Moran's $I$ values (Table 2). In 2013, clusters of regions with a high SFTS occurrence rate $(\mathrm{HH})$ appeared partially in the south-eastern region and on Jeju Island. Major $\mathrm{HH}$ clusters were observed in the eastern region of Gyeongbuk in 2014; the northern region of Gangwon-do in 2015 and 2016; the area of Gangwon and Gyeongbuk in 2017; the northern region of Gangwon in 2018; and Gangwon, Jeonbuk and Gyeongnam in 2019 (Figure 3).

We also found that the disease returns repeatedly to certain areas at a certain times. Such multi-frequency areas included seven areas within a cluster range for six years. They included Inje-gun, Sokcho-si, Yangyang-gun, Yeongyang-gun, Cheongsong-gun, Yeongcheon-si, and Buk-gu of Pohang-si. In some cases, the area of occurrence could expand every year.

Table 3 shows the region range with a remarkably high incidence of SFTS and the region most likely to have spatiotemporal clusters. In 2013, statistically significant clusters were observed from 1 May to 31 July $(R R=21.44, P<0.001)$. In 2014, 2017, 2018 and 2019, statistically significant clusters were observed from late May to early June to mid-October (2014: $\mathrm{RR}=19.00, \mathrm{P}<0.001$; 2017: $\mathrm{RR}=15.26, \mathrm{P}<0.001 ; 2018: \mathrm{R}=14.28, \mathrm{P}<0.00 ; 2019$ : 11.78, $\mathrm{P}<0.001$ ). In 2016, a significant cluster was observed for the shortest time during the study period from September 4 to October 29 $(\mathrm{RR}=28.73, \mathrm{P}<0.001)$.

Table 2. Summary of Global Moran's I results.

\begin{tabular}{lcccc}
\hline Year & Moran's I & Z score & P & Remark \\
2013 & 0.108 & 3.098 & $<0.01$ & Clustered \\
2014 & 0.106 & 3.021 & $<0.01$ & Clustered \\
\hline 2015 & 0.165 & 4.435 & $<0.01$ & Clustered \\
2016 & 0.339 & 9.134 & $<0.01$ & Clustered \\
\hline 2017 & 0.298 & 7.937 & $<0.01$ & Clustered \\
2018 & 0.263 & 6.940 & $<0.01$ & Clustered \\
\hline 2019 & 0.257 & 6.792 & $<0.01$ & Clustered \\
\hline
\end{tabular}
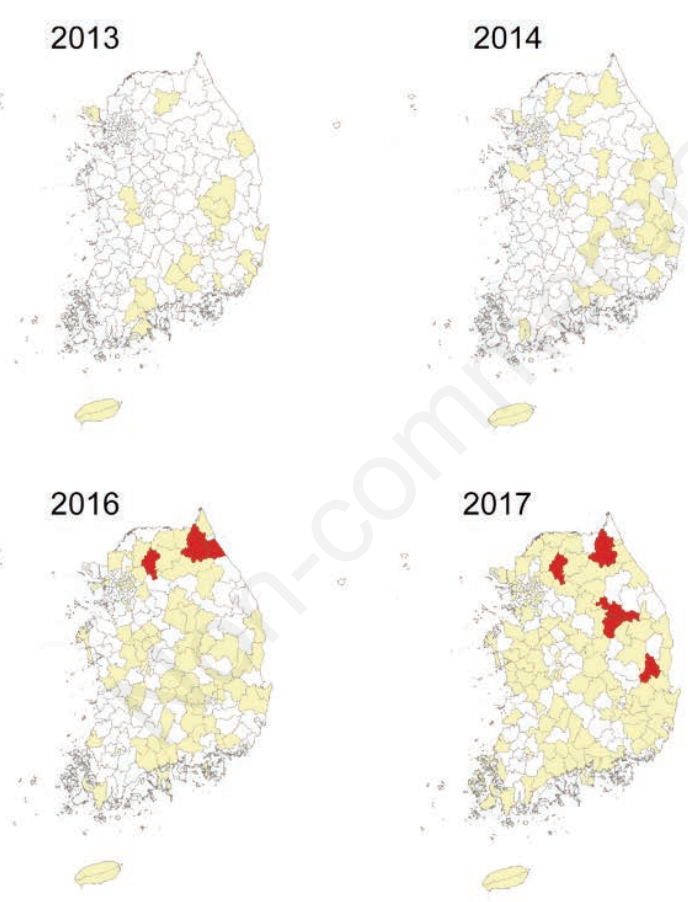

2019

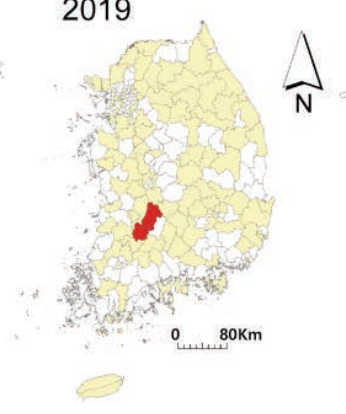

Incidence rate
2015
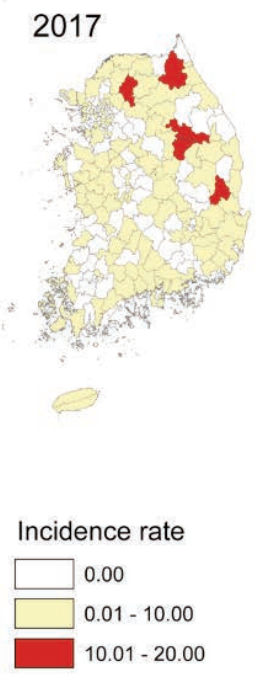

Figure 2. Cumulative incidence of the severe fever with thrombocytopenia syndrome in Republic of Korea 2013-2019. 


\section{Discussion}

Spatial analysis of high incidence of disease can be performed through various methods such as density estimation and spatial interpolation; however, these methods are limited by difficulty in processing time variables by treating disease information as a twodimensional and consequential factor without considering the time factors. Due to this limitation, a spatiotemporal cluster approach and a technique to calculate the risk were used.

The method of expressing and analysing disease outbreak information on a map is a basic method to analyse regional variation in disease incidence across public health-related fields (Kandwal et al., 2009). Understanding the geographic distribution of a disease can help identify high- or low-risk areas (Souza et al., 2019). Our results show that, in Korea, SFTS exhibits spatial autocorrelation (i.e. the disease occurs repeatedly at a certain time and

Table 3. SaTScan statistics for space-time clusters.

\begin{tabular}{|c|c|c|c|c|c|c|c|}
\hline Year & Cluster & Time frame & Zone (no.) & Cases obs. (no.) & Cases exp. (no.) & Relative risk & $\mathbf{P}$ \\
\hline 2013 & Most likely & 05/01-07/31 & 39 & 12 & 0.82 & 21.44 & $<0.0001$ \\
\hline 2014 & Most likely & $06 / 05-10 / 22$ & 44 & 22 & 1.86 & 19.00 & $<0.0001$ \\
\hline 2015 & Most likely & $08 / 28-11 / 05$ & 14 & 15 & 0.39 & 47.48 & $<0.0001$ \\
\hline 2016 & Most likely & $09 / 04-10 / 29$ & 45 & 48 & 2.32 & 28.73 & $<0.0001$ \\
\hline 2017 & Most likely & $06 / 05-10 / 22$ & 40 & 94 & 9.10 & 15.26 & $<0.0001$ \\
\hline 2018 & Most likely & 05/29-10/15 & 27 & 63 & 5.70 & 14.28 & $<0.0001$ \\
\hline 2019 & Most likely & 05/29-10/29 & 28 & 54 & 5.89 & 11.78 & $<0.0001$ \\
\hline
\end{tabular}
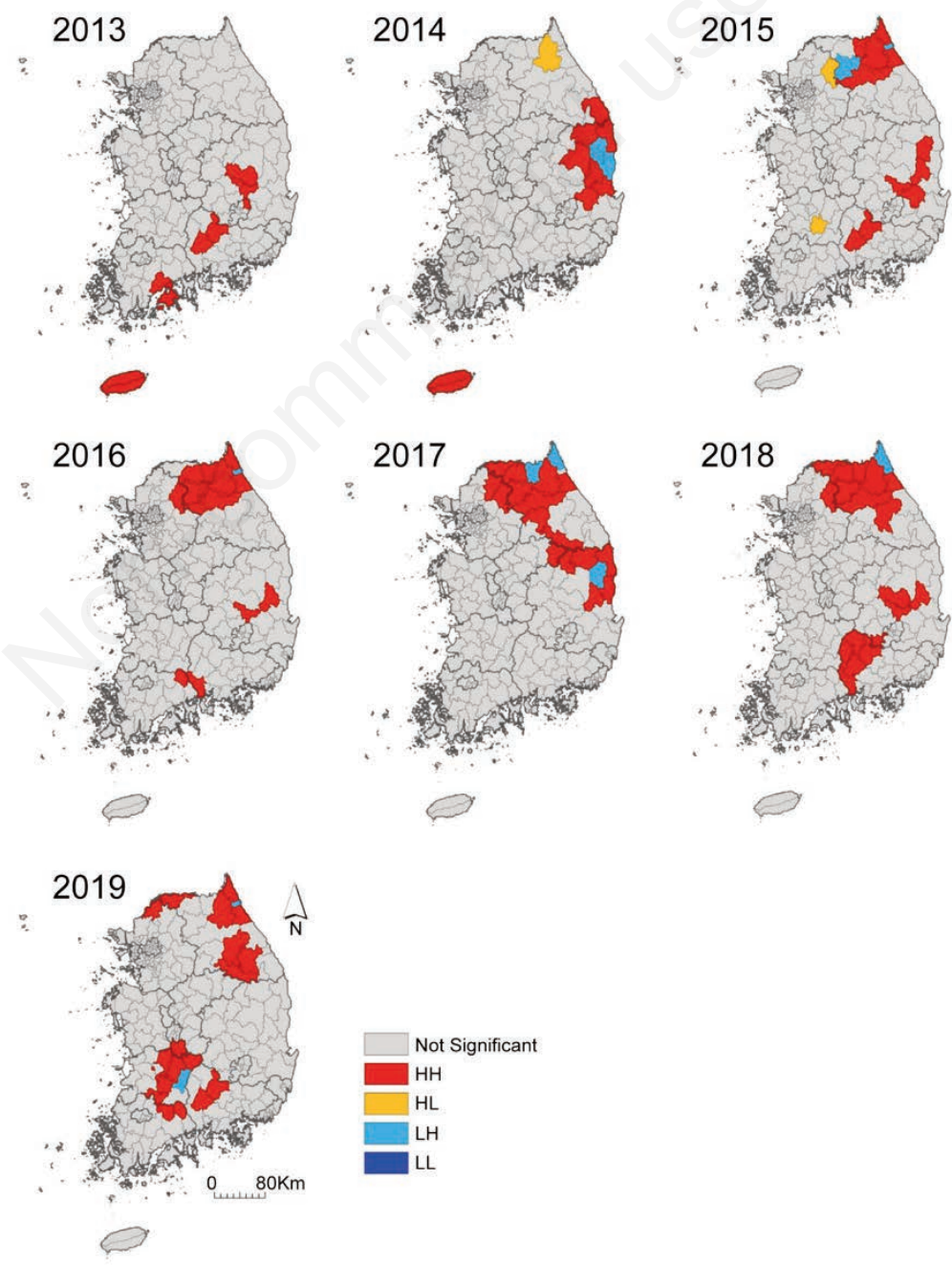

Figure 3. LISA cluster maps for the severe fever with severe fever thrombocytopenia syndrome 2013-2019. 
in a certain area). For example, the county units (gun) in each city were selected for regional cluster (LISA) analysis and the findings showed some areas to be vulnerable to such spatial multi-frequency SFTS clustering.

Identification of regional clusters may provide valuable epidemiological insights into SFTS bolster its surveillance in Republic of Korea. Clusters in areas with a high RR ratio were found to occur every year, and this pattern should be evaluated for potential risk factors. The addition of spatial scan statistics analysis to rapid statistical analysis during monitoring would help in the management of potentially at-risk areas (Desjardins et al., 2020). Thus, identifying areas with a high RR ratio for SFTS would help health authorities to monitor outbreaks in a timely manner and guide effective allocation of medical resources to manage disease outbreaks and prevent recurrence (Greenwood \& Reid, 2020).

The monthly occurrence of SFTS in Republic of Korea during the initial monitoring period was found to be concentrated in JulyAugust in the summer, while the time range expanded to early summer and autumn in recent years (2017-2018). This is in agreement with the finding by Miao et al. (2020) in China and several other Asian countries and supported by our SaTScan results of significant clusters with a high SFTS incidence between May and October in most years of observation. A study on SFTS in China suggests that the cause of the increasing incidence of SFTS could be due to a rise in infection opportunities and improved experience in the diagnosis of SFTS (Sun et al., 2017). This could be the explanation for the similar increasing trend in Republic of Korea as well. However, it could also be due to a higher susceptibility in those above 50 years of age as they amount to $91 \%$ of all patients and constitute the main age group at risk in Republic of Korea (Figure 1). The real reason could be underlying diseases and decreased immune function. As there is currently no vaccine available to prevent SFTS, it is important to observe precautionary measures (Liu et al., 2014). One should be aware of the time and region of the SFTS's temporal and spatial weakness.

A significant limitation of this study is that factors such as socioeconomic level, education level, occupation, geographic characteristics and weather characteristics, which can affect the occurrence pattern of SFTS patients, were not addressed. Further research that considers the effect of these factors on SFTS incidence is warranted.

\section{Conclusions}

The importance of spatiotemporal, epidemiological analysis in identifying SFTS cluster areas requiring targeted public health prevention was demonstrated. Although the cumulative incidence of SFTS in Republic of Korea showed an increasing trend with a territorial expansion, and there was a constant seasonal pattern over the last 7 years (2013-2019). These results should help establish effective plans for controlling further spread of SFTS.

\section{References}

Andresen MA, 2011. Estimating the probability of local crime clusters: the impact of immediate spatial neighbors. J Crim Justice 39:394-404.

Anselin L, 1995. Local indicators of spatial association-LISA. Wiley Online Library 27:93-115.

Desjardins MR, Hohl A, Delmelle EM, 2020. Rapid surveillance of COVID-19 in the United States using a prospective spacetime scan statistic: detecting and evaluating emerging clusters. Appl Geogr 118:102202.

Ding F, Zhang W, Wang L, Hu W, Soares Magalhaes RJ, Sun H, Zhou H, Sha S, Li S, Liu Q, Li Q, 2013. Epidemiologic features of severe fever with thrombocytopenia syndrome in China, 2011-2012. Clin Infect Dis 56:1682-3.

Greenwood KP, Reid SA, 2020. Clustering of cryptosporidiosis in Queensland, Australia, is not defined temporally or by spatial diversity. Int J Parasitol 50:209-16.

Guo CT, Lu QB, Ding SJ, Hu CY, Hu JG, Wo Y, Fan YD, Wang XJ, Qin SL, Cui N, Yang ZD, 2016. Epidemiological and clinical characteristics of severe fever with thrombocytopenia syndrome (SFTS) in China: an integrated data analysis. Epidemiol Infect 144:1345-54.

Han J, Lee M, 2016. Cancer cluster detection using scan statistic. J Korean Data Inf Sci Soc 27:1193-1201.

Kandwal R, Garg PK, Garg RD, 2009. Health GIS and HIV/AIDS studies: perspective and retrospective. J Biomed Inform 42:748-55.

KDCA, 2016. Recommendation for medical care guidelines for severe acute thrombocytopenia syndrome [in Korean]. [cited 2020]. Available from: http://www.kdca.go.kr/npt/biz/npp/portal/nppPblctDtaView.do?pblctDtaSeAt $=8$ \&pblctDtaSn $=820$

Kulldorff M, 2018. Software for the spatial and space-time scan statistics. Kulldorff and Harvard Medical School, Boston and Information Management Services Inc. (2018). SaTScan ${ }^{\mathrm{TM}_{\mathrm{V}} 9.6 \text {. Available from: http://www.satscan.org/ }}$ Accessed: 1 July 2018.

Liu K, Zhou H, Sun RX, Yao HW, Li Y, Wang LP, Mu D, Li XL, Yang Y, Gray GC, Cui N, 2015. A national assessment of the epidemiology of severe fever with thrombocytopenia syndrome, China. Sci Rep 5:9679.

Liu Q, He B, Huang SY, Wei F, Zhu XQ, 2014. Severe fever with thrombocytopenia syndrome, an emerging tick-borne zoonosis. Lancet Infect Dis 14:763-72.

Li Z, Hu J, Cui L, Hong Y, Liu J, Li P, Guo X, Liu W, Wang X, Qi $\mathrm{X}, \mathrm{Wu} \mathrm{B}, 2017$. Increased prevalence of severe fever with thrombocytopenia syndrome in Eastern China clustered with multiple genotypes and reasserted virus during 2010-2015. Sci Rep 7:6503.

McMullan LK, Folk SM, Kelly AJ, MacNeil A, Goldsmith CS, Metcalfe MG, Batten BC, Albariño CG, Zaki SR, Rollin PE, Nicholson WL, 2012. A new phlebovirus associated with severe febrile illness in Missouri. N Engl J Med 367:834-41.

Miao D, Dai K, Zhao GP, Li XL, Shi WQ, Zhang JS, Yang Y, Liu W, Fang LQ, 2020. Mapping the global potential transmission hotspots for severe fever with thrombocytopenia syndrome by machine learning methods. Emerg Microbes Infect 9:817-26.

Oh WS, Heo ST, Kim SH, Choi WJ, Han MG, Kim JY, 2014. Plasma exchange and ribavirin for rapidly progressive severe fever with thrombocytopenia syndrome. Int J Infect Dis 18:84-6.

Seo CW, Kim JH, Kim EY, Lee SE, Park HK, 2018. Epidemiological characteristics of patients with severe fever with thrombocytopenia syndrome in Republic of Korea in 2017. Public Health Weekly Report KCDC 11:698-706.

Souza EC, Santos ES, Rosa AM, Botelho C, 2019. Space-time scan for identification of risk areas for hospitalization of children due to asthma in Mato Grosso, Brazil. Rev Bras Epidemiol 22:e190019.

Sun J, Lu L, Wu H, Yang J, Ren J, Liu Q, 2017. The changing epidemiological characteristics of severe fever with thrombocytopenia syndrome in China, 2011-2016. Sci Rep 7:9236. 
Sun JM, Wu HX, Lu L, Liu Y, Mao ZY, Ren JP, Yao WW, Qu HH, Liu QY, 2020. Factors associated with spatial distribution of severe fever with thrombocytopenia syndrome. Sci Total Environ [Epub ahead of print].

Takahashi T, Maeda K, Suzuki T, Ishido A, Shigeoka T, Tominaga T, Kamei T, Honda M, Ninomiya D, Sakai T, Senba T, 2014. The first identification and retrospective study of severe fever with thrombocytopenia syndrome in Japan. J Infect Dis 209:816-27.

Yoo JR, Heo ST, 2018. Strategies against severe fever with thrombocytopenia syndrome increasing in Korea. Korean J Blood Transfus 29:117-29.

You E, Wang L, Zhang L, Wu J, Zhao K, Huang, F, 2020.
Epidemiological characteristics of severe fever with thrombocytopenia syndrome in Hefei of Anhui Province: a populationbased surveillance study from 2011 to 2018. Eur J Clin Microbiol Infect Dis [Epub ahead of print].

Yun SM, Park SJ, Kim YI, Park SW, Yu MA, Kwon HI, Kim EH, Yu KM, Jeong HW, Ryou J, Lee WJ, Jee Y, Lee JY, Choi YK, 2020. Genetic and pathogenic diversity of severe fever with thrombocytopenia syndrome virus (SFTSV) in South Korea. JCI Insight 5:e129531.

Yu XJ, Liang MF, Zhang SY, Liu Y, Li JD, Sun YL, Zhang L, Zhang QF, Popov VL, Li C, Qu J, 2011. Fever with thrombocytopenia associated with a novel bunyavirus in China. N Engl J Med 364:1523-32. 